\title{
Comparison of Oncological and Functional Results of Open and Robot- Assisted Laparoscopic Radical Cystectomy for Bladder Cancer
}

\author{
Resul Sobay, $\odot$ Ahmet Tahra, $\odot$ Uğur Tolga Şen, $\odot$ Ferhat Yakup Suçeken, $\odot$ Eyüp Veli Küçük, \\ Uğur Boylu \\ Department of Urology, Health Sciences University, Umraniye Training and Research Hospital, Istanbul, Turkey
}

\begin{abstract}
Introduction: To assess oncological and functional outcomes of two techniques for patients with bladder cancer who undergone open radical cystectomy (ORC) or robot- assisted radical cystectomy (RARC).

Methods: 47 patients who underwent ORC and RARC due to bladder cancer between 2009 and 2017 were evaluated. Preoperative, operative and postoperative follow-up data was recorded prospectively and analyzed retrospectively. Preoperative and postoperative data were compared using Student Paired t test and Mann-Whitney $U$ test. Surivival of the patients were analyzed using Kaplan-Meier test.

Results: 34 patients underwent ORC and 13 patients underwent RARC. The mean age of ORC and RARC groups were 61.7 and 62 , respectively ( $p=0.904$ ). The mean operating time (6 hours) in the ORC group was statistically significantly shorter than the RARC group (7 hours). Estimated blood loss was statistically significantly higher in the ORC group [350 (100-800)] than that in the RARC group [150 (100-400)] ( $p=0.001)$. The postoperative complication rates were similar between the two groups ( $p>0.05)$. There was no statistically significant difference between two groups in terms of hospital stay ( $p=0.596)$, rates of positive surgical marginand number of removed lymph nodes ( $p>0.05$ ). The mean overall survival time was 42.7 months in the ARS group and 51.3 months in the RARC group ( $p=0.893$ ).

Discussion and Conclusion: Robot- assisted radical cystectomy has comparable oncological outcomes to ORC. Although ORC has a shorter operating time, RARC has advantages in term of less blood loss and earlier start of oral feeding.

Keywords: Bladder cancer; open cystectomy; robot-assisted radical cystectomy.
\end{abstract}

$\mathrm{B}^{1}$ ladder cancer is the $7^{\text {th }}$ most common cancer among the male population worldwide. When both genders were evaluated in combination, bladder cancer the $11^{\text {th }}$ place. Globally, age-standardized mortality rates due to bladder cancer in 2012 were 3.2 for men, and 0.9 for women (100.000 person/year) ${ }^{[1]}$.

Approximately $25 \%$ of bladder cancers are muscle inva- sive at the time of diagnosis ${ }^{22]}$. Standard treatment for invasive bladder cancer is open radical cystectomy (ORC) [3]. High-risk and frequently recurring superficial tumors, high-grade CIS and T1 tumors resistant to BCG therapy, and widespread bladder tumors which can not be controlled by transurethral resection are also other indications for radical cystectomy ${ }^{[4,5]}$. 
Radical cystectomy and associated urinary diversion is one of the most radical surgical operations of urology practice due to higher perioperative mortality and morbidity rates. When the various series are examined, perioperative mortality ranging from $3-8 \%$ and perioperative complication rates reaching up to $60 \%$ can be seen $[6,7]$.

One of the minimally invasive surgical techniques commonly used in urology practice is robot- assisted surgery. It was first applied by Menon et al. ${ }^{[8]}$ in 2003 in a robotassisted radical cystectomy (RARC) series consisting of 17 cases, afterwards it has been applied in various centers. Advantages such as lesser blood loss, lower transfusion rate, faster recovery time, shorter hospital stay compared to open radical cystectomy as shown in various series ${ }^{[9-12]}$.

\section{Materials and Methods}

Patients who underwent ORC and RARC with the indication of bladder cancer between the years 2009 and 2017 at the Urology Clinic after approval from our hospital ethics committee were evaluated retrospectively. Patients with a short follow-up period of three months were excluded from the study. Preoperative, perioperative, postoperative clinical and oncologic data of patients were recorded prospectively and analyzed retrospectively. Operations were performed by a single surgeon.

Patients' demographic data, age, sex, BMI, ASA score, preoperative pathology, preoperative clinical stage, parameters related to operation, duration of operation, blood loss, duration of hospital stay, time to oral intake, preoperative and postoperative creatinine and albumin levels were evaluated. Fathological parameters as pathological stage, grade, surgical margin positivity, number of lymph nodes removed and number of positive lymph nodes, presence of primary prostatic adenocarcinoma, recurrences during oncologic follow-ups, and presence of metastasis were assessed. The latest information about the condition of the patients who were lost to follow-up were directly gathered by phone or from their close relatives.

The American Cancer Society TNM staging was used in the evaluation of preoperative clinical stage. According to follow-up protocol, the patients were monitored postoperatively at every 3 , months within the first 2 years, and every 6 months within the third year, then at yearly intervals.

Physical examination, biochemical tests, chest x-ray and ultrasound examinations were performed at follow-up visits. Apart from the routine assessments, advanced imaging modalities as CT or MRI were used for patients with suspect metastasis or recurrence. Thirty-four out of 35 patients who underwent ORC and followed up for more than 3 months, and 13 out of 15 patients who underwent RARS were included in the study.

\section{Preoperative Preparation}

Patients were hospitalized 3 days preoperatively. Intestinal preparation protocol was applied for 3 days. Low-molecular-weight heparin was administered one day before the operation.

\section{Surgical Technique used in Robot-Assisted Radical Cystectomy}

Under general anesthesia, nasogastric tube and $18 \mathrm{Fr}$ Foley catheter were inserted. Approximately $5 \mathrm{~cm}$ superior to the umbilicus in the midline, a $8-10 \mathrm{~mm}$ camera port was inserted through skin incision made with a scalpel, then skin and subcutaneous tissue were passed through. Pnomoperitoneum was created by entering into peritoneal cavity with a Veress needle. After the camera port was inserted, the patient was positioned at about 25 degreeTrendelenburg position.

Subsequently the other ports were placed under direct vision. The access ports of the two robotic arms were placed to the left and right of the rectus mauscle lateral to the umbilicus. Then the $4^{\text {th }}$ port was inserted through a point lateral to the right port and about $3 \mathrm{~cm}$ below the umbilical level. A 5 $\mathrm{cm}$ long assistant port with a caliber of $12 \mathrm{~mm}$ was placed on the left side between the camera port and the working port. After ureters were identified, they were dissected up to the ureterovesical junction. Then the ureters were clipped with the help of 2 hemoclips, and cut. Distal tips of ureters were cut, and sent for perioperative frozen section examination. The peritoneum over the seminal vesicles was then incised. The seminal vesicles were dissected and lateral parts were cut between hemoclips. Vas deferenses were transected. Denonvillier fascia was opened and the prostatic base was dissected up to urethrovesical junction. Lateral to the prostatic base vesical pedicles were ligated with the aid of a sealant (LigaSure Atlas ${ }^{\mathrm{TM}}$ ). Subsequently, the peritoneum was opened lateral to the medial umbilical ligaments.

Anterior abdominal wall was retracted, and exposed up to the external iliac artery, and Retzius space was entered.The endopelvic fascia was opened and the deep venous complex was ligated with absorbable $2 / 0$ sutures, and transected. The urethra was dissected and then clipped and transected. Cystoprostatectomy specimen was pleced in a $15 \mathrm{~mm}$ laparoscopic specimen bag. After lymph node dissection (extended lymph node dissection) up to the aortic bifurcation level, we proceeded with urinary diversion (Fig. 1). 


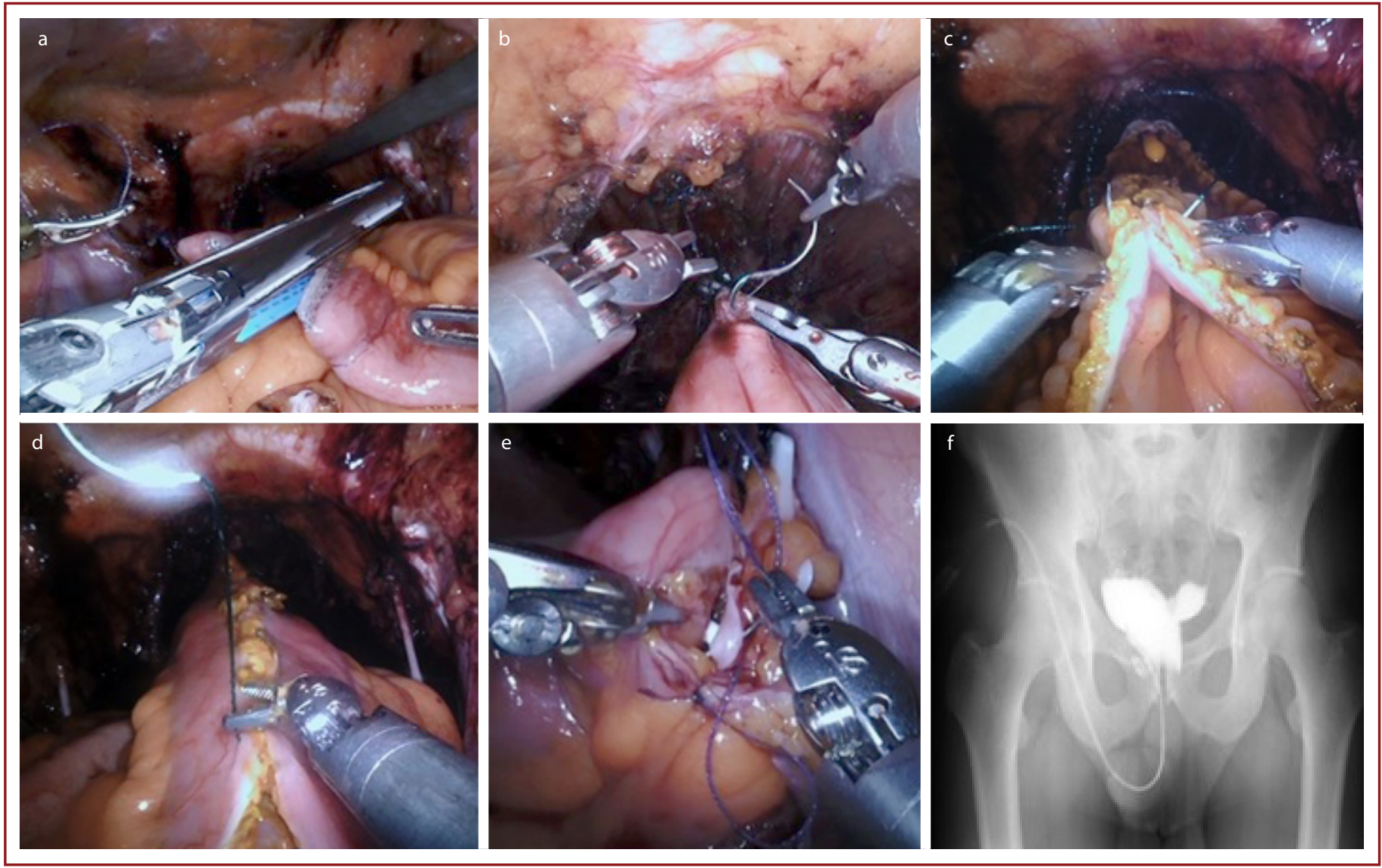

Figure 1. Ileal neobladder. (a) Transection of ileum using staplers. (b) lleo-Urethral Anastomosis. (c, d) Construction of Y-Neobladder Using Continuıous Sutures. (e) Uretero-Ileal anastomosis. (f) Postoperative X-ray appearance of Y-Neobladder.

\section{Robot-Assisted Radical Cystectomy in a Female Patient}

Classical anterior pelvic exenteration was performed in female patients. The infundibulopelvic ligaments and ovarian pedicles located in the posterior part of the bladder were dissected and the uterus and ovaries were freed. After identification of the uterine arteries, they were clamped with hemoclips, and transected. The uterovaginal junction was defined with the aid of a Reverdin retractor placed through the vagina. The vagina was incised and the anterior vaginal wall was included among the specimens that will be sent for histopathological examination. The vagina was closed with continuous sutures.

\section{Open Radical Cystectomy: Surgical Technique}

A 12-16 cm longitudinal median incision extending from superior part of the umbilicus to the symphysis pubis was made. Peritoneum was dissected away from both sides of the urachus towards the bladder. Radical cystectomy was performed as described in the literature.

\section{Urinary Diversion}

Open radical cystectomy and RARC were performed with ureterointestinal anastomosis using Bricker technique. In this technique, terminal ends of the ureters were spatulated and separately anastomosed using end-to-side technique to the intestinal segment with absorbable $4 / 0$ sutures.

\section{Robot-Assisted Orthotopic Urinary Diversion:}

A Y- neobladder was constructed in intracorporeal orthotopic urinary diversion. A $40 \mathrm{~cm}$-long ileal segment with its mesentery was isolated from $25 \mathrm{~cm}$ proximal to the ileocecal valve. Intestinal continuity was achieved with staplers. Once both ends of the isolated intestinal segment were closed, and its distal $30 \mathrm{~cm}$ was detubularized from its antimesenteric side.

The ureters were spatulated and anastomosed to the proximal afferent tubular intestinal segment using. Bricker technique, and $6 \mathrm{Fr}$ mono $\mathrm{J}$ catheters were placed into the ureters. The Y-shaped bowel was closed with continuous sutures to form a neobladder. Then, a urethral anastomosis was performed over the 16-18 Fr Foley catheter using the Van Velthoven method. 
Postoperative complications were evaluated using the Clavien-Dindo classification system, which was introduced by Clavien in 1992, and modified by Dindo and Clavien in 2004. Its results covering a 5 -year period were published in $2009^{[13]}$.

\section{Statistical Analysis}

For the statistical analyses, the IBM SPSS Statistics 22 (IBM SPSS, USA) package program was used to evaluate findings obtained in the study. Parameters with normal distribution were evaluated using Shapiro- Wilk test.

Student $t$ test was used for comparisons between both groups, Mann-Whitney $U$ test was used for comparisons between two groups of parameters that did not show normal distribution, while descriptive statistical methods (mean, standard deviation, frequency) were used for the evaluation of quantitative data.

Wilcoxon Signed-Rank test was used for intragroup comparisons of parameters with non-normal distribution. Fisher's Exact test, Fisher's Freeman Halton test and continuity (Yates) correction were used for comparison of qualitative data. Kaplan-Meier method and LogRank test were used for survival analysis. Statistical significance was assessed at $\mathrm{p}<0.05$ level.

\section{Results}

Thirty-four patients who underwent open radical cystectomy and 13 patients who underwent RARC were included

Table 1. Preoperative findings

\begin{tabular}{|c|c|c|c|}
\hline & ORC Group & RARC Group & $\mathbf{p}$ \\
\hline Number of Patients & 34 & 13 & \\
\hline $\begin{array}{l}\text { Age Median } \\
\text { (minimum-maximum) } \\
\text { (years) }\end{array}$ & $62(47-77)$ & $62(47-74)$ & 0.904 \\
\hline $\begin{array}{l}\text { BMI }\left(\mathrm{kg} / \mathrm{m}^{2}\right) \text { Median } \\
\text { (minimum-maximum) }\end{array}$ & $28(22-37)$ & $26(22-31)$ & 0.231 \\
\hline \multicolumn{4}{|l|}{ Gender (\%) } \\
\hline Male & $28(82.4)$ & $12(92.3)$ & 0.655 \\
\hline Female & $6(17.6)$ & $1(7.7)$ & \\
\hline \multicolumn{4}{|l|}{ ASA Score (\%) } \\
\hline 1 & $3(8.8)$ & 0 & 0.087 \\
\hline 2 & $23(67.6)$ & $13(100)$ & \\
\hline 3 & $8(23.5)$ & 0 & \\
\hline \multicolumn{4}{|l|}{ Preoperative intravesical } \\
\hline \multicolumn{4}{|l|}{ BCG instillation (\%) } \\
\hline No & $32(94.1)$ & $12(92.3)$ & 1 \\
\hline Yes & $2(5.9)$ & $1(7.7)$ & \\
\hline
\end{tabular}

in the study. When preoperative data of the patients were evaluated, 28 male and 6 female patients were included in the ORC group. One female, and twelve male patients were included in the RARC group.

The median age was 62 years in both groups. Statistically significant differences were not detected between the ORC and RARC groups in terms of distribution of age, BMI values, gender of the patients, and ASA scores ( $p>0.05)$. The findings are given in Table 1.

When the patients' perioperative surgical data were evaluated, total operative time of the ORC group was found to be statistically significantly shorter than that of the RARC group ( $p=0.001$ ).

It was observed that the median (minimum-maximum) values of the estimated blood loss in the ORC and RARC groups were 350 (100-800) $\mathrm{ml}$ and 150 (100-400) $\mathrm{ml}$, respectively. As is seen, statistically significantly greater amount of blood loss was observed in the ORC group $(p=0.001)$.

On the other hand, no statistically significant difference was found between ORC and RARC groups in terms of the need for perioperative transfusion ( $p>0.05$ ), while two (5.9\%) patients underwent additional surgery (nephrectomy) during ORC (Table 2).

Median (minimum-maximum) values for postoperative hospital stay in the ORC, and RARC groups were 15 (12-18), and 14 (11-19) days, respectively without statistically significant difference between both groups ( $p=0.596)$.

Median (minimum-maximum) values in the ORC, and RARC groups in terms of time to oral feeding were 4 (2-12) days, respectively. The transition time to oral feeding was statistically significantly shorter in the RARC group $(p=0.001)$ (Table 3).

There was no statistically significant change in postoper-

Table 2. Perioperative characteristics and need for additional surgery

\begin{tabular}{lccc}
\hline & ORC & RARC & p \\
\hline $\begin{array}{l}\text { Operative time (hours) } \\
\text { Median (minimum-maximum) }\end{array}$ & $6(5.5-7)$ & $7(7-8)$ & 0.001 \\
$\begin{array}{l}\text { Estimated Blood Loss (ml) } \\
\text { Median (minimum-maximum) }\end{array}$ & $350(100-800)$ & $150(100-400)$ & 0.001 \\
$\begin{array}{l}\text { Transfusion } \\
\quad \text { Yes }\end{array}$ & 20 & & \\
$\quad$ No & 14 & 4 & 0.163 \\
$\begin{array}{l}\text { Additional surgical } \\
\text { intervention (\%) }\end{array}$ & & 9 & \\
$\quad$ Yes & & & \\
$\quad$ No & $2(5.9)$ & $0(0)$ & \\
\hline
\end{tabular}


ative creatinine values when compared with preoperative creatinine values in both ORC and RARC groups ( $p>0.05$ ). Preoperative albumin values were examined to assess nutritional status of the patients, and a statistically significant difference was not found between ORC $[3.65(1.7-4,5) \mathrm{g} /$ $\mathrm{dL}]$ and RARC [3.7 (2.2-4.3) g/dL] groups ( $\mathrm{p}>0.05$ ) (Table 4).

Distribution of T1 (0 vs 15.4\%), T2a (29.4 vs $7.7 \%)$, T2b (2.9 vs $23.1 \%)$, T3a (11.8 vs $23.1 \%)$, T3b (14.7 vs $7.7 \%)$, and T4a (41.2 vs $23.1 \%$ ) in ORC, and RARC groups was as indicated. The pathological data of patients are summarized in Table 5. Complications were not observed in $64.7 \%$ of the patients in the open radical cystectomy group, while Clavien 3B (14.7\%), 3A (8.8\%), I (5.9\%), and 2 (5.9\%) complications were seen in respective percentages of the patients.

In $53.8 \%$ of the patients in the robot-assisted radical cystectomy any complication was not seen, while Clavien 3B (23.1\%), and I (23.1\%), complications were seen in respective percentages of the patients. Grade 1-2 were grouped among minor, and grade 3-5 in major complications. There were no statistically significant differences between the two groups in terms of major and minor complications ( $p>0.05)$.

As types of diversion were concerned, the patients in the open radical cystectomy group underwent extracorporeal ileal conduit $(n=30)$, and extracorporeal orthotopic neobladder $(n=4)$. However in the robot-assisted radical cystectomy group; the patients underwent extracorporeal ileal conduit $(n=5)$, extracorporeal orthotopic neobladder $(n=3)$, intracorporeal ileal conduit $(n=3)$ and had intracorporeal orthotopic neobladder $(n=2)$.

The mean overall survival time of the patients was 42.7 (SD \pm 6.57 ) months in the ORC group and 51.3 (SD \pm 11.4 ) months in the RARC group. There was no statistically signif-

Table 3. Postoperative hospital stay, and time to oral nutrition

\begin{tabular}{lccc}
\hline & ORC & RARC & P \\
\hline $\begin{array}{l}\text { Postoperative hospital stay (days) } \\
\text { Median (minimum-maximum) }\end{array}$ & & & \\
Time to Oral Nutrition (days) & $4(2-12)$ & $2(1-8)$ & 0.001 \\
Median (minimum-maximum) & & & \\
\hline
\end{tabular}

icant difference between the two groups in terms of mean overall survival times ( $p=0.893$ ) (Fig. 2).

\section{Discussion}

In the most Western countries, radical cystectomy and pelvic lymph node dissection is the standard treatment for localized muscle-invasive bladder tumors and non-muscle invasive bladder tumors carrying very high risk ${ }^{[3,14]}$. Radical cystectomy can be performed using open, laparoscopic and robot assisted surgical techniques.

Today, minimally invasive procedures are being performed more and more frequently, and have found a wider indication at this stage, laparoscopic and robot- assisted surgery have come into prominence. Robot-assisted surgery has advantages of providing three-dimensional image, optical magnification up to ten times, and opportunity of

Table 5. Pathological Findings

\begin{tabular}{|c|c|c|c|}
\hline & ORC & RARC & $\mathbf{p}$ \\
\hline \multicolumn{4}{|l|}{ pT (\%) } \\
\hline T1 & $0(0)$ & $2(15.4)$ & - \\
\hline $\mathrm{T} 2 \mathrm{a}$ & $10(29.4)$ & $1(7.7)$ & \\
\hline $\mathrm{T} 2 \mathrm{~b}$ & $1(2.9)$ & $3(23.1)$ & \\
\hline T3a & $4(11.8)$ & $3(23.1)$ & \\
\hline T3b & $5(14.7)$ & $1(7.7)$ & \\
\hline $\mathrm{T} 4 \mathrm{a}$ & $14(41.2)$ & $3(23.1)$ & \\
\hline \multicolumn{4}{|l|}{ pN (\%) } \\
\hline NO & $17(50)$ & $9(69.2)$ & - \\
\hline N1 & $8(23.5)$ & $0(0)$ & \\
\hline $\mathrm{N} 2$ & $7(20.6)$ & $4(30.8)$ & \\
\hline N3 & $2(5.9)$ & $0(0)$ & \\
\hline \multicolumn{4}{|l|}{ Positive surgical margin (\%) } \\
\hline Var & $2(5.9)$ & $0(0)$ & 1 \\
\hline Yok & $32(94.1)$ & $13(100)$ & \\
\hline Median (minimum-maximum) & $16(3-44)$ & $17(3-31)$ & 0.190 \\
\hline \multicolumn{4}{|l|}{$\begin{array}{l}\text { Number of extracted positive } \\
\text { lymph nodes median }\end{array}$} \\
\hline (minimum-maximum) & $0-5$ & $0-6$ & 0.237 \\
\hline \multicolumn{4}{|l|}{ Positive lymph Node (\%) } \\
\hline Present & $16(47.1)$ & $3(23.1)$ & 0.243 \\
\hline Absent & $18(52.9)$ & $10(76.9)$ & \\
\hline Primary prostatic adenocarcinoma & $12(35.3)$ & $1(7.7)$ & - \\
\hline
\end{tabular}

Table 4. Preoperative and postoperative creatinine and preoperative albumin values

\begin{tabular}{lcc}
\hline & ORC & RARC \\
\hline Preoperative Creatinine (mg/dl) Median (minimum-maximum) & $0.99(0.66-4)$ & $0.87(0.72-3.39)$ \\
Postoperative Creatinine (mg/dl) Median (minimum-maximum) & $1.07(0.72-3.25)$ & $1(0.7-2.57)$ \\
Preoperative Albümin (g/dl) Median (minimum-maximum) & $3.65(1.7-4.5)$ & $3.7(2.2-4.3)$ \\
\hline
\end{tabular}




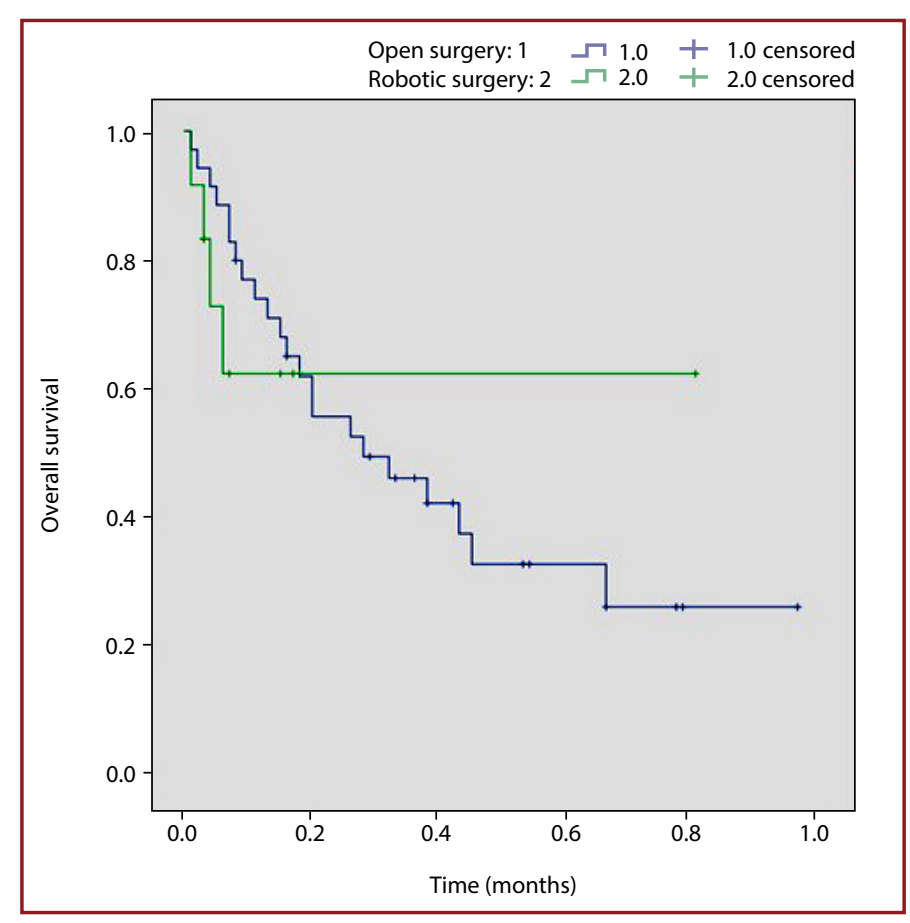

Figure 2. Average overall survival times of both groups.

movement in seven planes compared to the standard laparoscopy.

On the other hand, robot- assisted surgery is containdicated in patients who can not tolerate pneumoperitoneum and Trendelenburg position due to cardiac and pulmonary problems, and it is relatively contraindicated in patients with past history of extensive pelvic surgery which constitute its disadvantages compared to open surgery.

In our study, we aimed to compare the results of two minimally invasive techniques of ORC and RARC. Two groups were similar in terms of preoperative age, sex, body mass index, ASA score, and need for BCG instillation.

When we examined the current literature, we have found that in the study performed by the two high-volume centers in 2016, median operative times of 185 (165-222) and 330 (260-370) minutes were reported for ORC, and RARC, respectively ${ }^{[15]}$. In a recent randomized prospective study, the average operative time for RARC was found to be statistically significantly longer relative to ORC (360, vs 300 minutes, respectively ${ }^{[16]}$.

In our study, operative times for ORC, and RARC were found to be 360 (330-420) and 420 (410-480) minutes, respectively. Therefore, operative time was statistically significantly longer in the RARC group $(p=0.001)$. Operative time in the RARC group may be longer due to the difficulty in constructing intracorporeal diversion and the fact that preliminary cases of RARC were included in our study We think that the difference between the operative times will decrease in favor of RARC with increasing experience.

The estimated perioperative blood loss in our study was found to be statistically lower in the RARC group $(p=0.001)$. However, the perioperative transfusion rates were similar in both groups $(p=0.163)$. In a study by Gandaglia et al. ${ }^{[15]}$ estimated blood loss and perioperational transfusion rates were similar between the two groups. In a meta-analysis of 24 studies, evaluated by Soo et al. ${ }^{[17]}$ estimated blood loss and perioperative transfusion rates had been found to be statistically significantly lower in the RARC group.

In a meta-analysis performed by Soo et al. and in a study by Gandaglia et al. ${ }^{[15,17]}$ relatively shorter hospital stay was reported in favour of RARC In our study, hospital stay in the RARC group was statistically insignificantly shorter, in parallel with the literature. Furthermore, time to switching to oral feeding in the RARC group was statistically significantly shorter. We think that this is due to lesser intestinal manipulation during RARC.

In studies by Gandaglia et al. ${ }^{[15]}$ and Khan et al. ${ }^{[16]}$ any statistically significant difference between the two groups in terms of major and minor complications was not reported. In our study, any statistically significant difference was not observed between RARC and ORC groups in terms of major and minor complications.

In RARC, extended lymph node dissection up to the aortic bifurcation is recommended for staging and oncologic control ${ }^{[18]}$. In a meta-analysis, Soo et al. ${ }^{[17]}$ detected that the average number of 20 lymph nodes were removed in their case series, and reported no difference in terms of lymph node dissection compared to ORC. In the study conducted by the International Robotic Cystectomy Consortium, Hellenthal et al. reported that median number of 17.8 (0-68) lymph nodes were removed in RARC without any statistically significant difference when compared with ORC cases ${ }^{[19]}$. In our study, we detected that an average of 16 (3-44), and 17 (3-31) lymph nodes were removed in the ORC, and RARC groups, respectively. Parallel to the literature findings, the number of lymph nodes removed in our study was similar in both groups.

Surgical margin positivity is an important risk factor for the development of local recurrence and metastasis. In a single center study, 1589 patients were evaluated, with a positive surgical marginal rate of $4.2 \%$ and a 5 -year cancer-specific survival rate of $32 \%$ in patients with positive surgical margins ${ }^{[20]}$.

Gandaglia et al. ${ }^{[15]}$ reported that the rate of surgical margin positivity was $8.7 \%$ In the RARC group which was lower 
relative to ORC group without any statistically significant intergroup difference. In our study, there were no patients with surgical margin positivity in the RARC group. The rate of surgical margin positivity in the ORC group was 5.9\%, without any statistically significant intergroup difference.

When the literature was reviewed, it was reported that although most of the studies had a short follow-up period, there was no statistically significant difference between RARC and ORC in terms of overall survival ${ }^{[15-17]}$. Similarly, there was no statistically significant difference between the RARC and ORC groups in terms of the mean overall survival time in our study. As a limitation of our study, our group of robotic-assisted radical cystectomy consisted of preliminary cases of a series.

In addition, presence of bias in case selection because of the retrospective design of our study, and preference of ORC in patients with worse general health status are major disadvantages of ORC. On the other hand, application of these two techniques by the same surgeon in our clinic is an advantage of our study. Because of the retrospective nature of the majority of studies in the literature, prospective randomized studies are needed to demonstrate that RARC is equally effective compared with ORC.

For this reason, a multi-center randomized prospective study (RAZOR: Randomized Open vs. Robotic Cystectomy) was initiated in the USA in 2011. The 2-year results of this study, in which a total of 350 patients were randomized to RARC and ORC, were reported in 2017 AUA Conference. According to the reported results; a statistically significant difference could not be demonstrated between the RARC and ORC groups in terms of progression-free and overall survival rates ${ }^{[21]}$.

\section{Conclusion}

In the treatment of bladder cancer, RARC is a minimally invasive technique that provides lesser perioperative bleeding and earlier postoperative oral feeding than ORC technique. In the light of the current literature, RARC has oncologic and functional results comparable to ORC technique. In order to fully compare the two techniques, there is a need for randomized prospective studies with a higher number of patients with longer follow-up.

Ethics Committee Approval: The approval of the local Ethics Committee was obtained.

Peer-review: Externally peer-reviewed.

Authorship Contributions: Concept: R.S., U.B., E.V.K.; Design: R.S., A.T., F.Y.S.; Data Collection or Processing: R.S., U.T.Ş., F.Y.S.; Analysis or Interpretation: R.S., A.T., U.B.; Literature Search: R.S., U.T.Ş., U.B.; Writing: R.S., A.T., E.V.K., U.B.

Conflict of Interest: None declared.

Financial Disclosure: The authors declared that this study received no financial support.

\section{References}

1. Torre LA, Bray F, Siegel RL, Ferlay J, Lortet-Tieulent J, Jemal A. Global cancer statistics, 2012. CA Cancer J Clin. 2015;65:87108. [CrossRef]

2. DeSantis $C E$, Lin CC, Mariotto $A B$, Siegel RL, Stein KD, Kramer $\mathrm{JL}$, et al. Cancer treatment and survivorship statistics, 2014. CA Cancer J Clin 2014;64:252-71. [CrossRef]

3. Stein JP, Lieskovsky G, Cote R, Groshen S, Feng A-C, Boyd S, et al. Radical cystectomy in the treatment of invasive bladder cancer: long-term results in 1,054 patients. J Clin Oncol 2001;19:666-75. [CrossRef]

4. Sylvester RJ, van der Meijden AP, Oosterlinck W, Witjes JA, Bouffioux $C$, Denis $L$, et al. Predicting recurrence and progression in individual patients with stage Ta T1 bladder cancer using EORTC risk tables: a combined analysis of 2596 patients from seven EORTC trials. Eur Urol 2006;49:466-77. [CrossRef]

5. Fernandez-Gomez J, Madero R, Solsona E, Unda M, MartinezPiñeiro L, Gonzalez M, et al. Predicting nonmuscle invasive bladder cancer recurrence and progression in patients treated with bacillus Calmette-Guerin: the CUETO scoring model. J Urol 2009;182:2195-203. [CrossRef]

6. Stein JP, Skinner DG. Radical cystectomy for invasive bladder cancer: long-term results of a standard procedure. World J Urol 2006;24:296-304. [CrossRef]

7. Hautmann RE, de Petriconi RC, Volkmer BG. Lessons learned from 1,000 neobladders: the 90-day complication rate. J Urol 2010;184:990-4. [CrossRef]

8. Menon M, Hemal AK, Tewari A, Shrivastava A, Shoma AM, ElTabey NA, et al. Nerve-sparing robot-assisted radical cystoprostatectomy and urinary diversion. BJU Int 2003;92:232-6. [CrossRef]

9. Hemal AK, Abol-Enein H, Tewari A, Shrivastava A, Shoma AM, Ghoneim MA, et al. Robotic radical cystectomy and urinary diversion in the management of bladder cancer. Urol Clin North Am 2004;31:719-29, viii. [CrossRef]

10. Guru KA, Kim HL, Piacente PM, Mohler JL. Robot-assisted radical cystectomy and pelvic lymph node dissection: initial experience at Roswell Park Cancer Institute. Urology 2007;69:46974. [CrossRef]

11. Kang SG, Kang SH, Lee YG, Rha KH, Jeong BC, Ko YH, et al. Robot-assisted radical cystectomy and pelvic lymph node dissection: a multi-institutional study from Korea J Endourol 2010;24:1435-40.

12. Ahmed K, Khan SA, Hayn MH, Agarwal PK, Badani KK, Balbay $M D$, et al. Analysis of intracorporeal compared with extracorporeal urinary diversion after robot-assisted radical cystectomy: results from the International Robotic Cystectomy Consortium. Eur Urol 2014;65:340-7. [CrossRef] 
13. Clavien PA, Barkun J, de Oliveira ML, Vauthey JN, Dindo D, Schulick RD, et al. The Clavien-Dindo classification of surgical complications: five-year experience. Ann Surg 2009;250:18796. [CrossRef]

14. Shariat SF, Karakiewicz PI, Palapattu GS, Lotan Y, Rogers CG, Amiel GE, et al. Outcomes of radical cystectomy for transitional cell carcinoma of the bladder: a contemporary series from the Bladder Cancer Research Consortium. J Urol 2006;176:241422. [CrossRef]

15. Gandaglia G, Karl A, Novara G, de Groote R, Buchner A, D'Hondt $F$, et al. Perioperative and oncologic outcomes of robot-assisted vs. open radical cystectomy in bladder cancer patients: A comparison of two high-volume referral centers. Eur J Surg Oncol 2016;42:1736-43. [CrossRef]

16. Khan MS, Gan C, Ahmed K, Ismail AF, Watkins J, Summers $J A$, et al. A single-centre early phase randomised controlled three-arm trial of open, robotic, and laparoscopic radical cystectomy (CORAL). Eur Urol 2016;69:613-21. [CrossRef]

17. Son SK, Lee NR, Kang SH, Lee SH. Safety and Effectiveness of Robot-Assisted Versus Open Radical Cystectomy for Bladder
Cancer: A Systematic Review and Meta-Analysis. J Laparoendosc Adv Surg Tech A 2017;27:1109-20. [CrossRef]

18. Marshall SJ, Hayn MH, Stegemann AP, Agarwal PK, Badani KK, Balbay MD, et al. Impact of surgeon and volume on extended lymphadenectomy at the time of robot-assisted radical cystectomy: results from the International Robotic Cystectomy Consortium (IRCC). BJU Int 2013;111:1075-80. [CrossRef]

19. Hellenthal NJ, Hussain A, Andrews PE, Carpentier P, Castle E, Dasgupta $\mathrm{P}$, et al. Lymphadenectomy at the time of robot-assisted radical cystectomy: results from the International Robotic Cystectomy Consortium. BJU Int 2011;107:642-6. [CrossRef]

20. Dotan ZA, Kavanagh K, Yossepowitch O, Kaag M, Olgac S, Donat $M$, et al. Positive surgical margins in soft tissue following radical cystectomy for bladder cancer and cancer specific survival. J Urol 2007;178:2308-13. [CrossRef]

21. Smith ND, Castle EP, Gonzalgo ML, Svatek RS, Weizer AZ, Montgomery JS, et al. The RAZOR (randomized open vs robotic cystectomy) trial: study design and trial update. BJU Int 2015;115:198-205. [CrossRef] 